\title{
Controlled apneic tracheostomy in patients with coronavirus disease 2019 (COVID-19)
}

Kathleen D. Weiss, MD, ${ }^{\mathrm{a}}$ Antonio Coppolino III, MD, ${ }^{\mathrm{a}}$ Daniel C. Wiener, MD, ${ }^{\mathrm{a}}$ Ciaran McNamee, MD, ${ }^{\mathrm{a}}$ Robert Riviello, MD, ${ }^{\mathrm{b}} \mathrm{Ju}-\mathrm{Mei} \mathrm{Ng}, \mathrm{MD},{ }^{\mathrm{c}}$ Michael T. Jaklitsch, MD, ${ }^{\mathrm{a}}$ Margaret B. Marshall, MD, ${ }^{\mathrm{a}}$ and Matthew M. Rochefort, MD

\section{ABSTRACT}

Objective: To develop a team-based institutional infrastructure for navigating management of a novel disease, to determine a safe and effective approach for performing tracheostomies in patients with COVID-19 respiratory failure, and to review outcomes of patients and health care personnel following implementation of this approach.

Methods: An interdisciplinary Task Force was constructed to develop innovative strategies for management of a novel disease. A single-institution, prospective, nonrandomized cohort study was then conducted on patients with coronavirus disease 2019 (COVID-19) respiratory failure who underwent tracheostomy using an induced bedside apneic technique at a tertiary care academic institution between April 27, 2020, and June 30, 2020.

Results: In total, 28 patients underwent tracheostomy with induced apnea. The median lowest procedural oxygen saturation was $95 \%$. The median number of ventilated days following tracheostomy was 11 . There were 3 mortalities (11\%) due to sepsis and multiorgan failure; of 25 surviving patients, $100 \%$ were successfully discharged from the hospital and $76 \%$ are decannulated, with a median time of 26 days from tracheostomy to decannulation (range 12-57). There was no symptomatic disease transmission to health care personnel on the COVID-19 Tracheostomy Team.

Conclusions: Patients with respiratory failure from COVID-19 disease may benefit from tracheostomy. This can be completed effectively and safely without viral transmission to health care personnel. Performing tracheostomies earlier in the course of disease may expedite patient recovery and improve intensive care unit resource use. The creation of a collaborative Task Force is an effective strategic approach for management of novel disease. (JTCVS Techniques 2021;6:172-7)

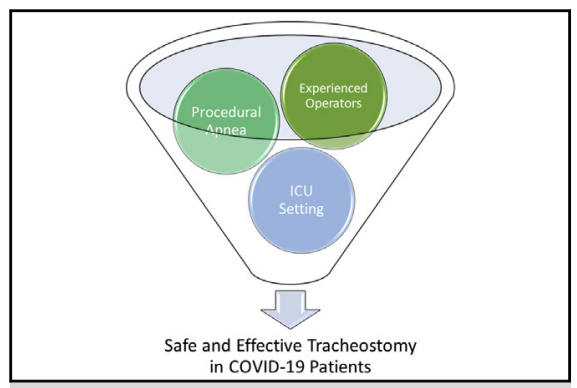

Components for a safe and effective apneic tracheostomy in patients with COVID-19.

CENTRAL MESSAGE

Patients with COVID-19 respiratory failure may benefit from tracheostomy, which can be completed effectively and safely and without symptomatic viral transmission to health care personnel.

\section{PERSPECTIVE}

Despite established benefits of tracheostomies, infectious concerns in the context of a pandemic are paramount. With induced apnea, tracheostomy can be done safely in patients with COVID-19 and with excellent clinical outcomes. It may be beneficial to perform tracheostomies earlier in the disease to expedite patient recovery and ventilator liberation and to optimize ICU resource use.

See Commentaries on pages 178 and 180.
The emergence of severe acute respiratory distress syndrome coronavirus 2 (SARS-CoV-2) and associated coronavirus disease 2019 (COVID-19) has rapidly evolved into an overwhelming global pandemic, affecting more than 185

From the ${ }^{\mathrm{a} D i v i s i o n}$ of Thoracic Surgery, Department of Surgery, ${ }^{\mathrm{b}}$ Division of Trauma, Burn, Surgical and Critical Care, Department of Surgery, and ${ }^{\mathrm{c} D e p a r t m e n t}$ of Anesthesiology, Brigham and Women's Hospital, Boston, Mass.

Received for publication Nov 9, 2020; accepted for publication Nov 18, 2020; available ahead of print Dec 7, 2020.

Address for reprints: Kathleen D. Weiss, MD, Division of Thoracic Surgery, Brigham and Women's Hospital, 75 Francis St, Boston, MA 02115 (E-mail: kweiss@bwh. harvard.edu). countries and leading to more than 590,000 deaths globally to date. ${ }^{1}$ Severe disease manifests with deterioration in respiratory status, frequently culminating in profound respiratory failure requiring prolonged mechanical ventilatory

\section{6-2507}

Copyright $(2020$ The Authors. Published by Elsevier Inc. on behalf of The American Association for Thoracic Surgery. This is an open access article under the CC BY-NCND license (http://creativecommons.org/licenses/by-nc-nd/4.0/).

https://doi.org/10.1016/j.xjtc.2020.11.016 


\section{Abbreviations and Acronyms \\ COVID-19= coronavirus disease 2019 \\ ECMO $=$ extracorporeal membrane oxygenation \\ ICU = intensive care unit \\ OR $\quad=$ operating room \\ PPE $\quad=$ personal protective equipment \\ RT $\quad=$ respiratory therapist \\ SARS-CoV-2 $=$ severe acute respiratory distress syndrome coronavirus 2}

support. ${ }^{2}$ Early data suggest that approximately $3 \%$ to $10 \%$ of patients with coronavirus ultimately require intubation during their hospitalization, ${ }^{2,3}$ with mortality rates of intubated patients as high as $70 \%{ }^{4,5}$ The acute influx of patients requiring intubation and ventilatory support has overwhelmed health care systems globally, leading to the urgent need to develop management strategies to optimize supportive care while appropriately balancing risk of transmissibility to health care workers and shortages of personal protective equipment (PPE).

Traditionally, patients with respiratory failure are candidates for tracheostomy if they require mechanical ventilatory support for 7 to 10 days or more. ${ }^{6} \mathrm{~A}$ tracheostomy facilitates ventilator weaning, reduces the need for sedation, enables earlier patient mobilization, and decreases the risk of laryngotracheal stenosis. ${ }^{7-9}$ In the context of the current pandemic, tracheostomy helps mitigate the potential hazards associated with therapies such as high-flow oxygen or noninvasive ventilation, which can generate potentially infectious aerosolized particles. ${ }^{10}$ In addition, in the context of limited intensive care unit (ICU) space and resources, tracheostomy facilitates patient transition from ICU-level care to inpatient rehabilitation, resulting in net increase of available ICU beds for other patients in need.

The risk of infectious aerosolization poses a challenge to traditional practices guiding patient selection, timing, and appropriateness for tracheostomy in patients with SARSCoV-2 respiratory failure. ${ }^{11}$ Current literature suggests that the overall prognosis for patients with COVID-19 in the ICU is poor. ${ }^{4}$ Uncertainties regarding disease progression, and data portending dismal outcomes, raise concerns that tracheostomy for patients with COVID-19 may be a clinically futile pursuit; in certain centers, tracheostomy has been eliminated from consideration for any patient with suspicion for active viral shedding. ${ }^{11,12}$ Aerosolized transmission raises significant infectious concerns for health care workers performing procedures on patients with COVID-19 and generates fear regarding the potential impact of frontline health care workers succumbing to the disease. ${ }^{13,14}$ Finally, in an era of acute resource shortages, the procedure requires consumption of PPE, which may be scarce in supply.

Determining the optimal technique, selection criteria, and timing for tracheostomies in patients with COVID-19 respiratory failure is at the forefront of clinical need. Here, we describe our early pandemic experience at a single, tertiary care academic institution performing tracheostomies in patients with respiratory failure secondary to SARS-CoV-2 infection. We present a framework for tracheostomy team organization, a modified procedural technique to minimize risk of viral transmission, and a discussion of patient as well as health care personnel outcomes.

\section{METHODS}

A single-institution, prospective, nonrandomized cohort study was conducted on patients with COVID-19 who underwent tracheostomy at the Brigham and Women's Hospital in Boston, Massachusetts, between April 27, 2020, and June 30, 2020. Data including demographic information, medical history, admission data, procedural details, duration of ventilator use, length of stay, and disposition information were clinically monitored and collected from electronic health records. The study was approved by the Partners Healthcare Institutional Review Board (institutional review board \#2014P002478; December 2, 2018), and informed consent was waived.

Onset of the pandemic prompted rapid assimilation of a highly organized COVID-19-specific infrastructure at the Brigham and Women's Hospital to develop a calculated and effective strategic approach to management of patients with SARS-CoV-2 infection. The "COVID-19 Task Force" leveraged multidisciplinary input to formulate a detailed methodology for safe and expeditious performance of tracheostomies in select patients with COVID-19 respiratory failure.

\section{The COVID Tracheostomy Team}

The institutional Task Force designated a subset of experienced and collaborative individuals to comprise the COVID-19 Tracheostomy Team. Approaches to management, procedural technique, and timing for COVID-19 tracheostomies were team-designed and implemented. Potential patients were reviewed, and the decision to proceed with tracheostomy prompted assignment of 5 essential team members for each case: 2 surgeons, 1 anesthesiologist, 1 nurse, and 1 operating room (OR) technician. Before any procedure, these 5 team members would briefly convene to review patient details and COVID-19-specific safety protocols established by the Task Force.

\section{Patient Selection}

Minimal physiologic criteria for patient selection included the ability to remain positioned supine for 30 minutes, a fraction of inspired oxygen requirement less than $60 \%$, and positive end-expiratory pressure less than or equal to $12 \mathrm{~mm} \mathrm{Hg}$. An intubation time in excess of 3 weeks was initially suggested for consideration of tracheostomy based on available evidence from other institutions; however, positive outcomes and few complications prompted relaxation of that timeline to approximately 2 weeks in certain circumstances.

\section{Location}

All tracheostomies were completed in negative-pressure rooms. Most were completed in the patient's ICU room; however, select cases were performed in the OR due to high-risk anatomy or because of concurrent, planned procedures. The ICU setting provided several benefits, namely, fewer disconnections from the ventilator and minimal exposure of hospital 
personnel (ie, no transport team or bystander exposure during patient travel). The ventilator control panel was positioned outside the room to enable control by a respiratory therapist (RT) without requiring direct patient contact with every adjustment, and to minimize the number of individuals in the room. A nurse and scrub technician were present for every tracheostomy as part of the assigned team; however, they remained outside the patient room for ICU-based procedures to reduce the risk of unnecessary viral exposure (Figure 1).

\section{Equipment}

A standardized set of equipment was used for each case, including a Blue Rhino tracheostomy kit (Cook Medical, Bloomington, Ind), an adult bronchoscope (Olympus America, Center Valley, Pa), an electrocautery source, sterile towels, a thyroid drape, PROLENE sutures (Ethicon, Bridgewater, NJ), and a Portex tracheostomy tube (cuffed, non-fenestrated) (Smiths Medical, Minneapolis, Minn). An open surgical instrument tray was also available for all cases, including those done percutaneously.

\section{Personal Protective Equipment}

Before room entry, personnel performing the procedure donned an N95 respirator mask, a standard surgical mask, a disposable scrub cap or bouffant, a Halyard isolation gown, surgical hood, surgical gown, closed eye protection, a face shield, inner and outer gloves, and knee-high shoe covers. A trained PPE observer was present to verify proper donning and doffing.

\section{Positioning and Technique}

Following initiation of paralysis and titration of sedation by the COVID19 team anesthesiologist, the RT initiated a set respiratory rate with $100 \%$ inspired oxygen to minimize the potential for desaturation during the procedure. Patients were positioned supine with a transverse rolled towel beneath the scapulae to augment neck extension. Following palpation of the anterior wall of the trachea and identification externally of the intended insertion site, the anesthesiologist directed RT to place the ventilator in standby mode. After initiation of apnea, the anesthesiologist disconnected the endotracheal tube from the ventilator circuit, advanced the bronchoscope through the endotracheal tube, deflated the endotracheal balloon, and withdrew the endotracheal tube under bronchoscopic view of the airway. Point pressure was visualized with the bronchoscope to verify the targeted location for placement. The Blue Rhino needle and angiocatheter were advanced under direct vision into the airway, and the remainder of the percutaneous dilational tracheostomy procedure was completed using a standard Seldinger technique. Immediately following cuff inflation and reconnection of the ventilator circuit as a closed system, the indication to resume ventilatory support was given.

\section{RESULTS}

From April 27, 2020, to June 30, 2020, 28 patients at the Brigham and Women's Hospital underwent elective tracheostomy due to respiratory failure secondary to COVID-19 disease (Table 1). The patient cohort ranged from 26 to 81 years of age (median 57 years), with a sex distribution of $71 \%$ male and $29 \%$ female. The predominance of male patients in this cohort is consistent with current literature demonstrating greater rates of severe disease among men. ${ }^{15}$ Underlying pulmonary conditions were present in $32 \%$ of patients. More than one half $(61 \%)$ were managed with prone positioning during their hospital stay, and $25 \%$ were patients who required venovenous extracorporeal membrane oxygenation (ECMO) support due to severity of disease. COVID-19 positivity was confirmed within 1 week of tracheostomy for $75 \%$ of the patients; for the remaining $25 \%$, COVID-19 positivity was confirmed at the time of admission.

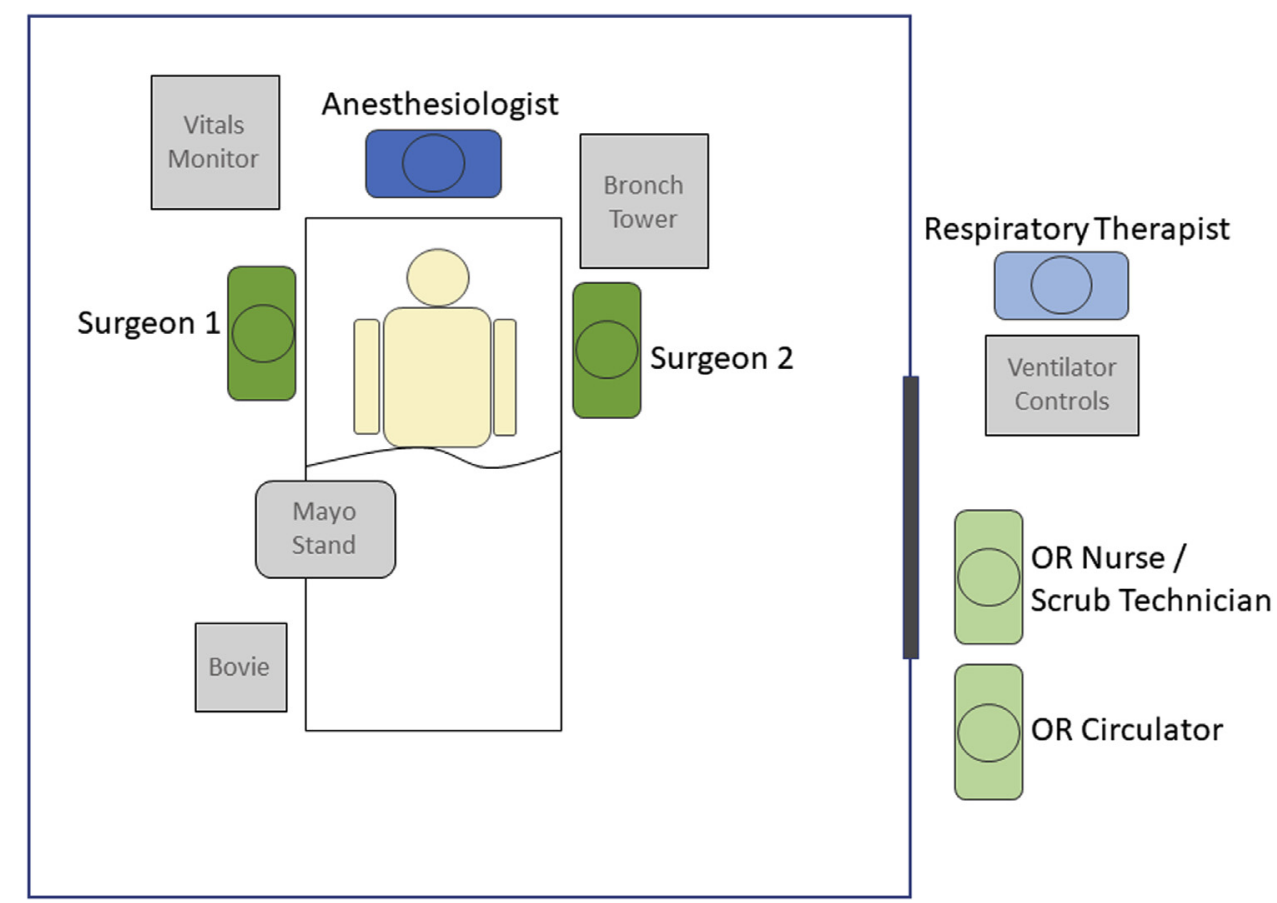

FIGURE 1. Setup for COVID-19 apneic tracheostomy in the intensive care unit setting, with the anesthesiologist at the head of the bed and the primary surgeon at the right side of the bed. OR, Operating room. 
TABLE 1. COVID-19 tracheostomy patient demographics

\begin{tabular}{lc}
\hline \multicolumn{1}{c}{ Characteristics } & Patients \\
\hline Median age, y (range) & $57(26-81)$ \\
Sex (\% male) & $71 \%$ \\
Median BMI (range) & $28.5(22.2-69.1)$ \\
Underlying pulmonary disease & $32 \%$ \\
Other medical comorbidity & \\
Immunocompromised & $7 \%$ \\
Cardiac disease & $21 \%$ \\
Hypertension & $43 \%$ \\
Diabetes & $43 \%$ \\
Chronic kidney disease & $11 \%$ \\
Transfers from outside hospitals & $75 \%$ \\
Proned & $61 \%$ \\
ECMO & $25 \%$ \\
\hline
\end{tabular}

$B M I$, Body mass index; $E C M O$, extracorporeal membrane oxygenation.

The timing of notable periprocedural events is detailed in Table 2. The median number of days from the time of initial intubation to the time of tracheostomy was 26 (range 12-48 days). All procedures were completed in negativepressure rooms. Of 28 cases, $25(89 \%)$ were performed in the patient's ICU room, and $27(96 \%)$ were completed via percutaneous approach. Three team members were present in the room for cases completed in the ICU, and 5 team members were present in the room for procedures completed in the OR due to case complexity, demonstrating an additional benefit in the form of risk reduction for exposure to health care workers when the procedure is performed in the ICU.

Aerosolization of secretions was minimized by performing airway entry and placement of the tracheostomy tube under deliberate and controlled apneic conditions. Paralysis was instituted to minimize response to airway manipulation to further decrease the risk of aerosolization. There were no physiologic concerns or clinically significant hemodynamic derangements during the period of apnea in $96 \%$ of cases. One patient with a body mass index of 69 , whose procedure was performed in the OR for anatomic concerns, became transiently bradycardic during the period of controlled apnea, with return to preapneic heart rate immediately upon resumption of ventilatory support. The median procedural apnea time was 3 minutes (range, 1-5 minutes), with a median lowest recorded procedural oxygen saturation of $95 \%$

TABLE 2. Timing of tracheostomy events

\begin{tabular}{ll}
\hline \multicolumn{1}{c}{ Event } & Median days (range) \\
\hline Pretracheostomy intubation & $26(12-48)$ \\
Tracheostomy to ventilator liberation & $11(2-42)$ \\
ICU stay following tracheostomy & $11(2-39)$ \\
Tracheostomy to decannulation & $26(12-57)$ \\
\hline
\end{tabular}

ICU, Intensive care unit.
TABLE 3. Tracheostomy procedural characteristics

\begin{tabular}{lc}
\hline \multicolumn{2}{c}{ Procedural Characteristic } \\
\hline Performed in ICU & $25(89 \%)$ \\
Percutaneous & $27(96 \%)$ \\
\hline Number of personnel in room (ICU vs OR) & $3 \mathrm{vs} 5$ \\
Median minutes of intraprocedural apnea (range) & $3(1-5)$ \\
\hline $\begin{array}{l}\text { Median lowest procedural oxygen } \\
\text { saturation (range) }\end{array}$ & $95 \%(62 \%-100 \%)$ \\
\hline$I C U$, Intensive care unit; $O R$, operating room.
\end{tabular}

while on $100 \%$ fraction of inspired oxygen during the procedure (Table 3).

The median number of days from tracheostomy to liberation from the ventilator was 11 (range, 2-42 days). There were 3 mortalities $(11 \%)$ due to sepsis and multisystem organ failure, 2 of whom were patients on ECMO; of the remaining 25 patients, $100 \%$ were successfully discharged from the hospital. At the time of publication, $76 \%$ have been decannulated, with a median time of tracheostomy to decannulation of 26 days (range, 12-57 days) (Table 4). There were zero instances of symptomatic disease transmission to health care personnel on the COVID-19 Tracheostomy Team.

Wound complications occurred in $6(21 \%)$ patients, including 3 patients with bleeding; all 3 instances of postoperative bleeding occurred in patients on ECMO support. Tracheostomy dislodgement occurred in $4(14 \%)$ patients.

\section{DISCUSSION}

Following reports of poor survival and risks of health care worker exposure, ${ }^{13}$ our institution and others originally advocated against elective aerosol-generating procedures for patients with COVID-19 disease. ${ }^{11}$ This study is one of the first to describe a modified tracheostomy technique, in conjunction with outcomes for patients and health care personnel following adoption of that technique, that supports elective tracheostomy. This technique, rapidly conceived and developed in response to an acute need, suggests that implementation of an experienced and dedicated team can provide early and effective care for high-risk patients with COVID-19. The technique itself is straightforward and requires equipment that is readily available at

TABLE 4. COVID-19 tracheostomy postoperative patient outcomes

\begin{tabular}{lc}
\hline \multicolumn{1}{c}{ Outcome } & $\mathbf{N = \mathbf { 2 8 }}$ \\
\hline Weaned off vent & 25 \\
Decannulated & 19 \\
Complications & 10 \\
Mortality & 3 \\
Discharged from the hospital (alive) & 25 \\
Home & 5 \\
\hline
\end{tabular}


most institutions, enabling facile widespread adoption of our approach. Percutaneous dilational tracheostomy has previously been demonstrated to be both quicker and less expensive than traditional open surgical tracheostomy. ${ }^{16}$ Our single-institution cohort of 28 patients is similar to other series in the current literature. ${ }^{17,18}$ We demonstrate excellent outcomes, with longer follow-up, which allows us to describe a more comprehensive assessment than other similarly-sized case series. ${ }^{17,18}$

The median number of days from the time of initial intubation to the time of tracheostomy was 26 days; however, the median duration to successful wean off the ventilator was only 11 days. Excluding mortalities, $100 \%$ were weaned off the ventilator, $100 \%$ of patients were discharged from the hospital, and $76 \%$ of patients were decannulated. These data suggest that the tracheostomy may promote more efficient ventilator weaning by means of decreased sedation, better airway hygiene, and increased ability to participate in physical therapy. Performing an earlier tracheostomy after 10 to 14 days of intubation could potentially result in shorter overall ventilator requirements. This has significant potential implications not only for augmenting patient recovery but also for improving ICU resource use.

Patients tolerated the brief period of induced apnea without notable complications and without developing significant hypoxia. The use of 2 experienced surgeons for each procedure minimizes procedural duration and apnea time. Performing the tracheostomy insertion under bronchoscopic guidance reduces the risk of inadvertent injury to the posterior membranous portion of the trachea by providing direct visualization of the airway. Importantly, there were zero instances of symptomatic viral transmission from patients undergoing tracheostomy to health care personnel on the COVID-19 Tracheostomy Team. The data support the safety and efficacy of this approach for the patient, as well as appropriate risk mitigation for those performing the procedure. Wound complications occurred in 6 patients $(21 \%)$, and inadvertent tracheostomy dislodgement occurred in $14 \%$, both of which are markedly greater than expected. Postprocedure stoma care presented an unanticipated challenge as it was subject to-and possibly compromised by-pandemic-specific safety measures, namely, those focused on minimizing direct patient contact to mitigate risk of transmission to hospital staff. Although it is fundamentally important to prioritize the safety of frontline workers, we suspect the resultant decrease in postprocedure face-to-face patient encounters led to less frequent and fewer total stoma evaluations postprocedurally. There was, consequently, greater risk for delayed identification of problems such as infection, local skin trauma, tracheostomy tube migration, or bleeding.

Our mortality rate of $10.7 \%$ is similar to the $7 \%$ described by Angel and colleagues ${ }^{18}$ and the $11.3 \%$ described by Chao and colleagues ${ }^{17}$; however, approximately $40 \%$ of the patients in both of those studies were still requiring positive pressure ventilatory support at the time of publication. At the time of this submission, all surviving patients have been liberated from the ventilator. Our outcomes suggest that patients with COVID-19 still actively requiring ECMO support may harbor a greater risk of significant morbidity and mortality associated with tracheostomy, and further assessment of ability to wean off ECMO support before proceeding with tracheostomy may be warranted.

There are several limitations with this study. For one, the experience and outcomes derive from a single center. Patient selection was not randomized, and there is potential for outcome bias inherent in selecting patients for the procedure who have already demonstrated relatively long survival on the ventilator. Extended follow-up data on patient outcomes are somewhat limited in favor of prioritizing early dissemination of information; however, we feel that this study represents the most comprehensive review of early outcomes of patients who underwent tracheostomy for COVID-19 infection. Criteria for patient selection and specific procedural methodologies may need to evolve as further research elucidates the intricacies of disease pathophysiology, and with advancement of treatment options and updated management strategies.

\section{CONCLUSIONS}

Accumulating and analyzing data and outcomes is paramount for establishing effective patterns and practices to inform and guide COVID-19 clinical care moving forward. This is one of the first studies to report specific strategies for risk mitigation when performing tracheostomies for patients with COVID-19, in conjunction with reporting a relatively large cohort of patient outcomes after implementation of those strategies. Our experience demonstrates that patients with respiratory failure secondary to COVID-19 disease benefit from tracheostomy, and that this procedure can be completed safely and without viral transmission to health care personnel. It may be beneficial to perform tracheostomies earlier in the clinical course of the disease to promote expedited patient recovery and ventilator liberation and to optimize ICU resource use. An organized, team-based infrastructure is advantageous in development of modified techniques for management of novel disease and facilitates a methodologic approach for successful delivery of advances in clinical care.

\section{Conflict of Interest Statement}

The authors reported no conflicts of interest.

The Journal policy requires editors and reviewers to disclose conflicts of interest and to decline handling or reviewing manuscripts for which they may have a conflict 
of interest. The editors and reviewers of this article have no conflicts of interest.

\section{References}

1. Center for Systems Science and Engineering. Coronavirus Covid-19 global cases by the Center for Systems Science and Engineering (CSSE) at Johns Hopkins University. Available at: https://coronavirus.jhu.edu/map. Accessed July 17, 2020.

2. Wu Z, McGoogan JM. Characteristics of and important lessons from the coronavirus disease 2019 (Covid-19) outbreak in China: summary of a report of 72314 cases from the Chinese center for disease control and prevention. JAMA. 2020; 323:1239-42.

3. Meng L, Qiu H, Wan L, Ai Y, Xue Z, Guo Q, et al. Intubation and ventilation amid the Covid-19 outbreak: Wuhan's experience. Anesthesiology. 2020;132: 1317-32.

4. Wu C, Chen X, Cai Y, Xia J, Zhou X, Xu S, et al. Risk factors associated with acute respiratory distress syndrome and death in patients with coronavirus disease 2019 pneumonia in Wuhan, China. JAMA Intern Med. 2020;180:934-43.

5. Zhou F, Yu T, Du R, Fan G, Liu Y, Liu Z, et al. Clinical course and risk factors for mortality of adult inpatients with Covid-19 in Wuhan, China: a retrospective cohort study. Lancet. 2020;395:1054-62.

6. Liu CC, Livingstone D, Dixon E, Dort JC. Early versus late tracheostomy: a systematic review and meta-analysis. Otolaryngol Head Neck Surg. 2015;152: 219-27.

7. Hosokawa K, Nishimura M, Egi M, Vincent JL. Timing of tracheotomy in ICU patients: a systematic review of randomized controlled trials. Crit Care. 2015;19: 424.

8. Koshkareva Y, Gaughan JP, Soliman AM. Risk factors for adult laryngotracheal stenosis: a review of 74 cases. Ann Otol Rhino Laryngol. 2007;116:206-10.
9. Griffiths J, Barber VS, Morgan L, Young JD. Systematic review and metaanalysis of studies of the timing of tracheostomy in adult patients undergoing artificial ventilation. BMJ. 2005;330:1243.

10. Tran K, Cimon K, Severn M, Pessoa-Silva CL, Conly J. Aerosol generating procedures and risk of transmission of acute respiratory infections to healthcare workers: a systematic review. PLoS One. 2012;7:e35797.

11. Shiba T, Ghazizadeh S, Chhetri D, St John M, Long J. Tracheostomy considerations during the Covid-19 pandemic. OTO Open. 2020;4:2473974X20922528.

12. Smith D, Montagne J, Raices M, Dietrich A, Bisso IC, Las Heras M, et al. Tracheostomy in the intensive care unit: guidelines during Covid-19 worldwide pandemic. Am J Otolaryngol. 2020;41:102578.

13. Haen P, Caruhel JB, Laversanne S, Cordier PY. Corona-steps for tracheotomy in Covid-19 patients: a staff-safe method for airway management. Oral Oncol. 2020;105:104729.

14. Adams JG, Walls RM. Supporting the health care workforce during the Covid-19 global epidemic. JAMA. 2020;323:1439-40.

15. Jin JM, Bai P, He W, Wu F, Liu XF, Han DM, et al. Gender differences in patients with Covid-19: focus on severity and mortality. Front Public Health 2020;8:152.

16. Freeman BD, Isabella K, Cobb JP, Boyle WA III, Schmieg RE Jr, Kolleff MH, et al. A prospective, randomized study comparing percutaneous with surgical tracheostomy in critically ill patients. Crit Care Med. 2001;29:926-30.

17. Chao TN, Braslow BM, Martin ND, Chalian AA, Atkins J, Haas AR, et al Tracheotomy in ventilated patients with Covid-19. Ann Surg. 2020;272:e30-2.

18. Angel L, Kon ZN, Chang SH, Rafeq S, Palasamudram Shekar S, Mitzman B et al. Novel percutaneous tracheostomy for critically ill patients with Covid-19. Ann Thor Surg. 2020;110:1006-11.

Key Words: COVID-19, SARS-CoV-2, coronavirus, pandemic, tracheostomy 